\title{
Relationships between vertical mixing and photoadaptation of phytoplankton: similarity criteria
}

\author{
M. R. Lewis ${ }^{1,2}$, J. J. Cullen ${ }^{2, *}$ and T. Platt ${ }^{2}$ \\ 1 Department of Biology, Dalhousie University, Halifax, Nova Scotia, Canada \\ 2 Marine Ecology Laboratory, Bedford Institute of Oceanography, Dartmouth, Nova Scotia, Canada
}

\begin{abstract}
In their natural environment, phytoplankton are exposed to fluctuations in incident irradiance due to vertical displacements in the water column induced by turbulent fluid motion. Physiological adaptation to these fluctuations results in variation in a number of measurable quantities (e.g. parameters of P-I curves, fluorescence yield, chemical composition), each of which have different time-scales of adaptation. A reaction-diffusion model is analyzed to determine the physical conditions under which a given physiological adaptation would be of ecological importance and thus may show variation in the photoadaptive variable with depth in a mixing layer. Two non-dimensional numbers control the dynamics. $\mathrm{K}_{\mathrm{v}} / \ell^{2} \gamma$ expresses the ratio of the mixing rate to the photoadaptation rate $\left(\mathrm{K}_{v}\right.$ is the eddy diffusion coefficient, $\ell$ is the mixing length, and $\gamma$ is the photoadaptation rate constant for given variable). $k_{s} \ell$ is the ratio of the mixing length scale to the optical length scale $\left(k_{\mathrm{s}}\right.$ is the attenuation coefficient for scalar irradiance). When $\mathrm{K}_{\mathrm{v}} / \ell^{2} \gamma$ is low and $\mathrm{k}_{\mathrm{s}} \ell$ is high, photoadaptation proceeds fast enough to create variations in depth in the photoadaptive variable and conversely, when $\mathrm{K}_{\mathrm{v}} / \ell^{2} \gamma$ is high and $\mathrm{k}_{\mathrm{s}} \ell$ is low, turbulent fluid motion dissipates any depth variation. By observing the variation in depth of several photoadaptive variables, each with a different photoadaptation time-scale, the mixing rate of the water can be estimated. Data from laboratory and field experiments are used to illustrate applications.
\end{abstract}

\section{INTRODUCTION}

In their natural environment, phytoplankton experience changes in irradiance that cover a broad range of time scales. Some of these can be ascribed to systematic causes, such as the diurnal and seasonal cycles in solar elevation; others we have to treat as stochastic events, even though the cause is known, for example changes in the weather (Dera and Olszewski, 1967), focussing of surface light by waves (Dera and Gordon, 1968), or turbulent transport of cells through the vertical light gradient (Platt and Gallegos, 1980; Falkowski and Wirick, 1981; Rivkin et al. 1982; Woods and Onken, 1982; Falkowski, in press).

The metabolic machinery of a phytoplankton cell is complex, and there is no reason to suppose that all of

\footnotetext{
- Present address: University of Texas, Port Aransas Marine Laboratory, Port Aransas, Texas 78373, USA
}

its component parts could adjust to changes in irradiance at the same rate. We expect therefore that a continued and directed change in irradiance would lead to a progression of compensations in various indices of phytoplankton metabolism, each measurable change corresponding to a process with a longer characteristic response time (cf. Harris, 1980a).

If the water column were perfectly stable and if surface irradiance were constant, all the metabolic processes of the phytoplankton at each depth would adjust to the ambient light at that depth. Of course, any vertical mixing tends to disturb this ideal of perfect adaptation. Because different indices of metabolism readjust at different rates, the possibility exists that the vertical mixing rate could be deduced from the vertical distribution of measurable metabolic properties. Here we develop the necessary theoretical background and give some examples of the application to both laboratory and field populations of phytoplankton. 


\section{CONCEPTUAL MODEL OF VERTICAL MIXING AND PHOTOADAPTATION}

In a vertical light gradient, the tendency for phytoplankton at different depths to adjust their photosynthetic characteristics to the local light level is opposed by the tendency for turbulence to erode this differentiation and bring the water column to a state that is physiologically, as well as physically homogenous. We immediately anticipate that the relative importance of the two opposing processes will be in large part determined by the relative magnitudes of the characteristic time scales of photoadaptation and turbulence. The ecological importance of algal photoadaptation within mixing layers has been investigated experimentally several times; the equivocal conclusions that can be reached reflect the uncertainty in matching the photoadaptation scale to the dominant time scale of light variations induced by fluid motion (Table 1). As can be seen, simulated mixing times span five orders of magnitude. A primary goal of our model approach is to determine the optical and fluid dynamical conditions where algal light intensity adaptation would be ecologically important.

The biological adjustment to light intensity (photoadaptation) is reflected in a number of photoadaptive parameters that are able to respond to changes in irradiance. Examples of photoadaptive parameters are: the in vivo fluorescence yield of chlorophyll a (Kiefer, 1973; Heaney, 1978; Vincent, 1979, 1980; Harris, $1980 \mathrm{~b})$, the chemical composition of phytoplankton (Gibson, 1978; Laws and Bannister, 1980; Falkowski and Wirick, 1981), ratios of photosynthetic pigments (Brooks, 1964; Falkowski, 1980; Prézelin and Matlick, 1980), photosynthetic capacity (Steemann Nielsen and Hansen, 1959; Steemann Nielsen and Jorgensen, 1968; Beardall and Morris, 1976; Falkowski, 1981), and in the broad sense, algal growth rate (Jitts et al., 1964; Paasche, 1968; Beardall and Morris, 1976; Hitchcock,
1980). Photoadaptive parameters do not all respond to changes in irradiance at the same rate however: photoinhibition of fluorescence is expressed on time scales of seconds to minutes (Loftus and Seliger, 1975; Vincent, 1979; Harris, 1980b; Abbott et al., 1982), but carbon-to-chlorophyll ratio or photosynthetic capacity change over several hours (Beardall and Morris, 1976; Owens et al., 1980; Marra, 1980).

Previous studies of photoadaptation and vertical mixing have discussed explicitly only one photoadaptive process or one photoadaptive rate at a time (Steemann-Nielsen and Hansen, 1959; Tilzer and Goldman, 1978; Falkowski and Wirick, 1981; but see Gibson, 1978; Harris, 1980b). Thus, the data previously presented were interpreted to suggest only an upper or lower bound for the mixing time of the surface layer. We propose that a suite of photoadaptive parameters can be measured simultaneously in a vertical profile; those photoadaptive parameters with characteristic rates of change faster than the temporal scale for vertical mixing will vary with depth, those that adapt more slowly than the phytoplankton are mixed will be adapted to a vertically homogeneous average of the fluctuations of irradiance experienced by phytoplankton in the layer. Thus both upper and lower bounds can be set to the vertical mixing rate.

We propose a simple model to compare the relative magnitudes of the time scales of photoadaptation with the time-scales of irradiance fluctuations experienced by passively transported particles (phytoplankton) during vertical mixing. The distributions with depth of various photoadaptive parameters are predicted in an Eulerian reference frame: the results can be checked by measurements at sea. Errors could arise if the contributions to the average photoadaptive variable by the individual phytoplankton are nonadditive; however in principle, the Eulerian equations can be transformed into a Lagrangian coordinate system if so desired (Hill, 1976), and higher order statistical moments calculated.

Table 1. Simulations of algal response to light fluctuations due to vertical mixing

\begin{tabular}{|c|c|c|c|}
\hline Type of simulation & Water movements considered ${ }^{1}$ & Mixing time-scale ${ }^{2}$ & Source \\
\hline Experimental & Directed & $\approx 0.1 \mathrm{~h}$ & Harris and Lott (1973) \\
\hline Experimental & Directed & $0.05 \mathrm{~h}$ & Jewson and Wood (1975) \\
\hline Experimental & Directed & $\approx 3 \mathrm{~h}$ & Kremer and Nixon (1978) \\
\hline Experimental & Directed & $1-1.5 \mathrm{~h}$ & Marra $(1978 a, b)$ \\
\hline Mathematical & Diffusive & $\approx 3.0 \mathrm{~d}$ & Platt and Gallegos (1980) \\
\hline Mathematical & Diffusive & $4.6-460 \mathrm{~d}$ & Falkowski and Wirick (1981) \\
\hline \multirow[t]{2}{*}{ Experimental } & Directed & $0.14-1.3 \mathrm{~h}$ & Gallegos and Platt (1982) \\
\hline & Diffusive & $4.3-64 h$ & \\
\hline
\end{tabular}


Consider a water column in which algal cells are uniformly distributed with depth. When the local irradiance varies, certain metabolic properties of the cells may adjust or vary as well in response. Let $\Gamma$ be a property of the phytoplankton that responds to changes in irradiance. The local rate of change of $\Gamma$ is

$$
\frac{\partial \Gamma}{\partial t}=f(I, t)
$$

where the function $f$ is to be defined.

In the case where $\Gamma$ is the chlorophyll concentration per cell, Falkowski (1980) and Falkowski and Wirick (1981) have proposed the following empirical functional form for $\mathrm{f}$ :

$$
\mathrm{f}(\mathrm{I}, \mathrm{t})=\gamma(\bar{\Gamma}(\mathrm{I})-\Gamma(\mathrm{I}, \mathrm{t}))=\gamma(\Delta \Gamma)
$$

where $\Delta \Gamma$ is the difference between $\Gamma$ at time $\mathrm{t}$ and some steady-state value $\bar{\Gamma}$ defined by the local incident irradiance. The rate constant, $\gamma\left(\mathrm{T}^{-1}\right)$, defines the rate of change.

Observed changes in $\Gamma$ at a given depth arise not only from local changes due to photoadaptation, but also because cells with perhaps different values of $\Gamma$ are mixed into and out of the depth interval.

$$
\frac{\partial \Gamma(z)}{\partial t}=\gamma(\Delta \Gamma)+\frac{\partial}{\partial z} K_{v} \frac{\partial \Gamma(z)}{\partial z}
$$

where $K_{v}$ = vertical eddy diffusion coefficient; $z$ (taken positive downwards) = depth. As a first approximation we assume $K_{\mathrm{v}}$ to be constant. We then re-express each original variable as the product of an appropriate scaling factor and a dimensionless variable (denoted by an asterisk) (cf. Wroblewski et al., 1975; Platt, 1982):

$$
\begin{aligned}
& \mathrm{z}=\ell \mathrm{z}^{*} \\
& \mathrm{t}=\tau \mathrm{t}^{*} \\
& \Gamma(\mathrm{z}, \mathrm{t})=\bar{\Gamma}(\mathrm{o}) \Gamma^{*} \\
& \Delta \Gamma(\mathrm{z}, \mathrm{t})=[\bar{\Gamma}(\mathrm{o})-\bar{\Gamma}(\ell)] \triangle \Gamma^{*}
\end{aligned}
$$

where $\bar{\Gamma}(0)=$ steady-state value of $\Gamma$ at the surface $(z=0) ; \bar{\Gamma}(\ell)=$ steady-state value at the bottom of the mixed layer at depth $\ell ; \tau=$ a time scale to be determined. The scale in the fourth equation is not independent of that in the third; it differs by implicit inclusion of the optical length scale in $\bar{\Gamma}(\ell)$ which scales $\bar{\Gamma}(z)$.

We furthermore insist initially that the scale for $\vec{\Gamma}$ (and $\Gamma$ by definition) be such that $\bar{\Gamma}(0)=1$ and $\bar{\Gamma}(\infty)$ be 0 . The surface irradiance is thus constant. Consequences of relaxation of this assumption are addressed later. Making the substitutions and rearranging,

$$
\frac{1}{\tau} \frac{\partial \Gamma^{*}}{\partial \mathrm{t}^{*}}=\gamma(\bar{\Gamma}(\mathrm{o})-\bar{\Gamma}(\ell)) \Delta \Gamma^{*}+\frac{\mathrm{K}_{v}}{\ell^{2}} \frac{\partial^{2} \Gamma^{*}}{\partial \mathrm{z}^{* 2}}
$$

We can set $\tau$ to either the time-scale for photoadaptation, $\gamma^{-1}(\bar{\Gamma}(0)-\bar{\Gamma}(\ell))^{-1}$, or to the turbulent time scale $\ell^{2} \mathrm{~K}_{\mathrm{v}}{ }^{-1}$. The relative magnitudes of the time scales determine which process will dominate changes in $\Gamma$ at a given depth. If both are of equal importance

$$
\frac{\mathrm{K}_{\mathrm{v}}}{\ell^{2}} \approx \gamma(\bar{\Gamma}(\mathrm{o})-\bar{\Gamma}(\ell)]
$$

For this result to be useful, we need to specify $\bar{\Gamma}(\mathrm{z})$. For example, although we do not insist on this form, we can let $\bar{\Gamma}(z)$ be proportional to local light intensity such that

$$
\bar{\Gamma}(z)=\bar{\Gamma}(0) e^{-k_{s} z}
$$

where $k_{\mathrm{s}}$ attenuation coefficient for scalar irradiance. In this case, Equation 6 becomes,

$$
\frac{\mathrm{K}_{\mathrm{v}}}{\gamma \ell^{2}} \approx 1-\exp \left(-\mathrm{k}_{\mathrm{s}} \ell\right)
$$

This is an important result since it divides all situations into 2 regions based on the magnitudes of the dimensionless groups $\mathrm{K}_{\mathrm{v}} / \gamma \ell^{2}$ and $\mathrm{k}_{\mathrm{s}} \ell$ : in one, a region where photoadaptation dominates and in the other, a region where turbulent fluid motion dominates (Fig. 1). When

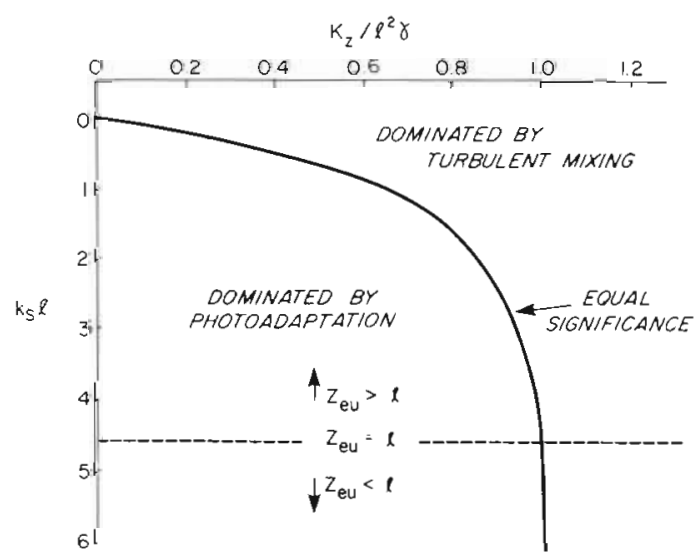

Fig. 1. Non-dimensional solution to Equation (3). The line divides the parameter space into a region where photoadaptation dominates and a region where turbulent diffusion dominates

$\mathrm{K}_{\mathrm{v}} / \gamma \ell^{2}$ is low (when the photoadaptation rate exceeds the rate of turbulent dissipation) and $\mathrm{k}_{\mathrm{s}} \ell$ is high (when the mean irradiance level is low and the irradiance gradient across the mixed layer is high) photoadaptation produces depth variations in $\Gamma$ faster than they can be broken down by turbulent exchange. On the other hand, when the irradiance gradient is low ( $k_{s} \ell$ is low) and the turbulent rate is higher than the photoadaptive rate $\left(\mathrm{K}_{\mathrm{v}} / \gamma \ell^{2}\right.$ is high), variations with depth in the photoadaptive variable are dissipated.

The dimensional approach is useful as we have shown, but the solutions should be regarded as an order of magnitude approximation. We have solved (3) explicitly using the formulation in (7) to describe $\bar{\Gamma}(\mathrm{z})$, subject to reflecting boundary conditions at the surface 
and bottom of the mixed layer. The steady-state solution is

$$
\begin{aligned}
& \frac{\Gamma(z)}{\bar{\Gamma}(0)}=\left[1-\frac{k_{v}}{r \ell^{2}}\left(k_{s} \ell\right)^{2}\right]-1\left[\exp \left(-k_{s} z\right)+\right.
\end{aligned}
$$

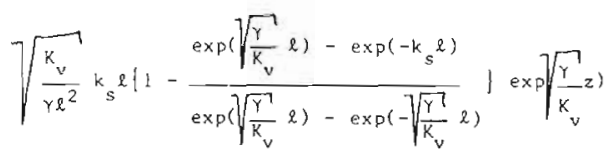

$$
\begin{aligned}
& \left|\sqrt{\frac{K_{v}}{\gamma \ell^{2}}} k_{s} 2\left\{\frac{\exp \left(\sqrt{\frac{\gamma}{K_{v}}} \ell\right)-\exp \left(-k_{s} \ell\right)}{\exp \left(\sqrt{\frac{\gamma}{K_{v}}} \ell\right)-\exp \left(\sqrt{\frac{Y}{K_{v}}} \ell\right)}\right\} \exp \left(-\sqrt{\frac{\gamma}{K_{v}}} z\right)\right|
\end{aligned}
$$

To compare the explicit solution to that derived solely on dimensional grounds, we have contoured the difference between the value of $\Gamma(\mathrm{z}) / \bar{\Gamma}(\mathrm{o})$ taken at surface and that at depth $\mathrm{z}=\ell$ for various values of the 2 dimensionless groups (Fig. 2). Our basic conclusion is borne out; when the photoadaptive rate process is high relative to the dissipative rate and the gradient in irradiance is high, more variation with depth results. It is also apparent that the solution is a function of the 2 groups, $\mathrm{K}_{\mathrm{v}} / \gamma \ell^{2}$ and $\mathrm{k}_{\mathrm{s}} \ell$. For example, if we let $\mathrm{K}_{\mathrm{v}}$ go to zero, Equation (9) reduces to the formula for $\Gamma$ under constant irradiance (Eq. 7). If we allow $\mathrm{K}_{\mathrm{v}}$ to become large relative to $\gamma \ell^{2}$, then the exponential terms cancel each other such that uniform conditions with depth result.

We have also solved (3) for the time-dependent case, subject to the additional condition that $\Gamma$ is uniformly distributed with depth at time zero (e.g. as a result of nocturnal convection). The solution is rather complex and its inclusion in its entirety here would not substantially add to the arguments. Suffice it to say that the efolding time scale associated with the damping of the transient is, to first order,

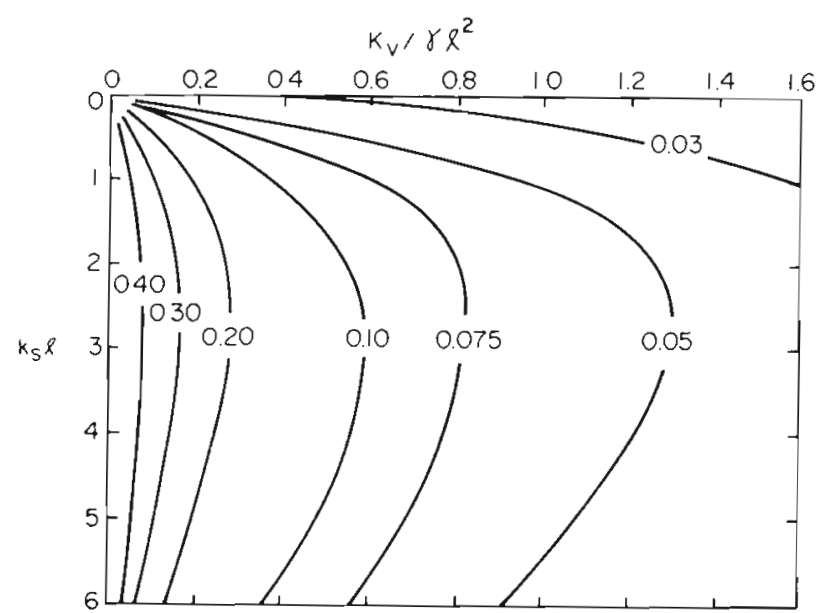

Fig. 2. Analytical solution to Equation (3). Isopleths indicate the normalized magnitude of the difference between the value of the photoadaptive parameter at the surface and at depth $=\ell$. Maximum difference is 1.0

$$
T \approx\left[\frac{\mathrm{K}_{\mathrm{v}} \pi^{2}}{\ell^{2}}+\gamma\right]^{-1}
$$

i.e. for a photoadaptive process with a characteristic time-scale of $\simeq 1 \mathrm{~h}$, an eddy diffusivity of $0.001 \mathrm{~m}^{2} \mathrm{~s}^{-1}$ and a mixed layer depth of $10 \mathrm{~m}$, the time required to approach the steady-state is of order $45 \mathrm{~min}$.

Our conclusions are not substantially altered for other formulations of the incident irradiance dependency of the photoadaptive variable. Falkowski and Wirick (1981) proposed a logarithmic relationship such that the dependence on depth is linear. The effect of this substitution is to straighten the line dividing the regions of photoadaptation and vertical mixing. Using their parameter values and a constant surface irradiance equal to the noon intensity we compute, as in $(8)$,

$$
\frac{\mathrm{K}_{\mathrm{v}}}{\gamma \ell^{2}}=0.215 \mathrm{k}_{\mathrm{s}} \ell
$$

for $\mathrm{k}_{\mathrm{s}} \ell<4.65$ and

$$
\frac{\mathrm{K}_{v}}{\gamma \ell^{2}}=1
$$

for $\mathrm{k}_{\mathrm{s}} \ell>4.65$.

We can further generalize Equation (3) by allowing the incident irradiance at depth to vary with time,

$$
\frac{\partial \Gamma(z)}{\partial t}=r i \frac{1}{T} \sum_{n=-\infty}^{\infty} A_{n} e^{1 \omega} n^{c} l e^{-k s^{2}}-\eta \Gamma(z)+\frac{\partial}{\partial z} K_{x} \frac{\partial \Gamma(z)}{\partial z}
$$

where $\frac{1}{T} \sum_{n=\infty}^{\infty} A_{n} e^{i \omega_{s} t}=$ sum of the Fourier terms for a fluctuating surface irradiance; $A_{n}=$ Fourier coefficient for the $n^{\text {th }}$ mode; $\omega_{n}=$ frequency. Again, we can apply dimensional reasoning and look at the magnitude of the non-dimensional group $\omega_{n} / \gamma$. If this number is large, the fluctuating frequencies are much higher than the rate of adaption; the cells would tend to average the fluctuations. If $\omega_{\mathrm{n}} / \gamma$ is small, the cells would track the low frequency oscillations since they can adjust their physiology much faster than the incident irradiance changes. The interesting case is when $\omega_{\mathrm{n}}=\gamma$. In this case, the physiological system is being forced at a frequency equivalent to the natural frequency of adaptation. It has been hypothesized that algal cells have evolved different physiological adaptation mechanisms with time-scales similar to those of the larger amplitude forcing frequencies to adapt to an inherently fluctuating environment (Harris, 1980a) and examples of such adaptive mechanisms have been observed (Prézelin and Sweeney, 1977; Demers and Legendre, 1981; Auclair et al., 1982).

We can employ similar reasoning to evaluate other terms which may be of importance. For example, there has been some interest in the influence of the vertical transport of algal cells in the helical vortices of Langmuir cells (Jewson and Wood, 1975; Marra, 1978b; Vincent, 1980). If the root mean square velocity associ- 
ated with Langmuir cells is $\bar{w}$, then the appropriate time-scale for this type of circulation is $\ell / \overline{\mathrm{w}}$, where $\ell=$ vertical extent of the Langmuir cells. The magnitude of $\ell / \bar{w}$ must then be weighed against the other time scales to warrant its inclusion in a given dynamical equation.

\section{DISCUSSION}

It has been shown that the interaction between photoadaptation and vertical mixing in determining the vertical distribution of biological parameters can be defined in terms of $\gamma$, the rate of photoadaptation, $\mathbf{k}_{\mathrm{s}}$ irradiance attenuation coefficient, $\ell$, the mixed layer depth, and $\mathrm{K}_{v}$, the coefficient of vertical eddy diffusion. Because $\ell, k_{s}$, and $\gamma$ can be measured in the field, it should be possible, in principle, to estimate timescales associated with vertical mixing by measuring the vertical distributions of photoadaptive parameters: of the photoadaptive parameters that are uniform with depth, the one with the fastest rate of change $\left(\Gamma_{1}\right)$ defines the lower bound of $\mathrm{K}_{\mathrm{v}}$. Using our formulation of $\Gamma$ (z) (Eq. 7) then,

$$
\mathrm{K}_{\mathrm{v}}>\ell^{2} \gamma_{1}\left(1-\mathrm{e}^{-\mathrm{k}_{\mathrm{s}} \varepsilon}\right)
$$

where $\gamma_{1}$ is the photoadaptive rate of $\Gamma_{1}$. The slowestadapting parameter of those that vary with depth $\left(\Gamma_{2}\right.$; photoadaptive rate $=\gamma_{2}$ ) specifies the upper bound of $K_{v}$ :

$$
\mathrm{K}_{v}<\ell^{2} \gamma_{2}\left(1-\mathrm{e}^{-\mathrm{k}_{s} \ell}\right)
$$

The equations can be modified to determine a characteristic vertical velocity associated with Langmuir circulation:

$$
\begin{aligned}
& \bar{w}>\ell \gamma_{1}\left(1-\mathrm{e}^{-k_{3} \ell}\right) \\
& \bar{w}<\ell \gamma_{2}\left(1-\mathrm{e}^{-k_{s} \ell}\right) .
\end{aligned}
$$

Similar calculations of the eddy diffusion coefficient have been made using the distribution with depth of diurnal changes in temperature (reviewed in Pollard, 1977).

The use of the analogy with Fickian diffusion to parameterize the turbulent velocity field is hardly defensible on physical grounds; nonetheless it continues to be useful in dynamic models of the upper ocean (e.g. Zaneveld et al., 1981). The use of the distribution of photoadaptative parameters to infer mixing rates parameterized as $\ell^{2} / \mathrm{K}_{\mathrm{v}}$ implies that the measurement of the biological properties be averaged on at least the same spatial scale implicit in the use of a bulk number such as $K_{v}$ to average the small-scale turbulence. This limits the usefulness since the spatial scale over which the biological properties are averaged is of the order of a meter, the scale associated with a bottle cast. An exception is the measurement of continuous fluorescence parameters (Lorenzen, 1966; Denman and Platt, 1975; Cullen and Renger, 1979; Derenbach et al., 1979; Abbott et al., 1982).

Valid application of the model requires reliable estimates of the photoadaptive rate, $\gamma_{i}$, for each photoadaptive parameter, $\Gamma_{1}$. Furthermore, a wide range of photoadaptive rates should be represented. Can their time-scales be specified?

Available results from a number of studies indicate that the application of our model may be feasible. Several strong photoadaptive responses have been studied, measured in vertical profiles, and in many cases related to water movements. For example, the increase of chlorophyll fluorescence upon addition of the photosynthetic inhibitor dichlorophenyl dimethylurea-(DCMU) can be very responsive to changes of light intensity and has a characteristic time scale for change of less than 1 to several h or more, depending in part on the means of measurement (Harris, 1980b; Vincent, 1980). Vertical profiles of the DCMU-induced fluorescence response of natural phytoplankton have been related to the intensity of wind mixing (Harris, $1980 \mathrm{~b}$ ), and have been used to suggest the physiological consequences of Langmuir circulation (Vincent, 1980). Likewise, photosynthesis-irradiance curves have been determined for samples from different depths and the previous light-history of the phytoplankton inferred (Steemann Nielsen and Hansen, 1959; Talling, 1966; Tilzer and Goldman, 1978; Falkowski, 1980; Platt et al., 1982, Gallegos et al., in press); for changes in photosynthetic performance, the characteristic time scale for change can be less than 12 h up to several d (Steemann Nielsen et. al. 1962; Yentsch and Lee, 1966; Beardall and Morris, 1976; Prézelin and Matlick, 1980; Marra, 1980), but it appears to be faster than the algal generation time. Rates for photoadaptation have also been measured for pigment ratios (Brooks, 1964; Falkowski, in press, Prézelin and Matlick, 1980), chlorophyll content (Brooks, 1964; Prézelin and Matlick, 1980) and carbohydrate content (Gibson, 1978). However, a large set of photoadaptive parameters and the corresponding photoadaptive rates have not yet been determined simultaneously on any one population.

Preliminary results from our laboratory suggest that for the marine diatom Thalassiosira pseudonana (Clone $3 \mathrm{H}$ ), physiological adjustment to changes in light intensity is manifested in several easily measurable quantities with a broad range of definable rates of change. Further experimental details and results are reported elsewhere (Cullen et al., in prep.). Cultures were grown under continuous illumination of 20,100 , and $2400 \mu$ Ein $\mathrm{m}^{-2} \mathrm{~s}^{-1}$ at $21^{\circ} \mathrm{C}$. The time course of photoadaptation was followed for each of the six possible shifts between irradiance levels. Experiments 


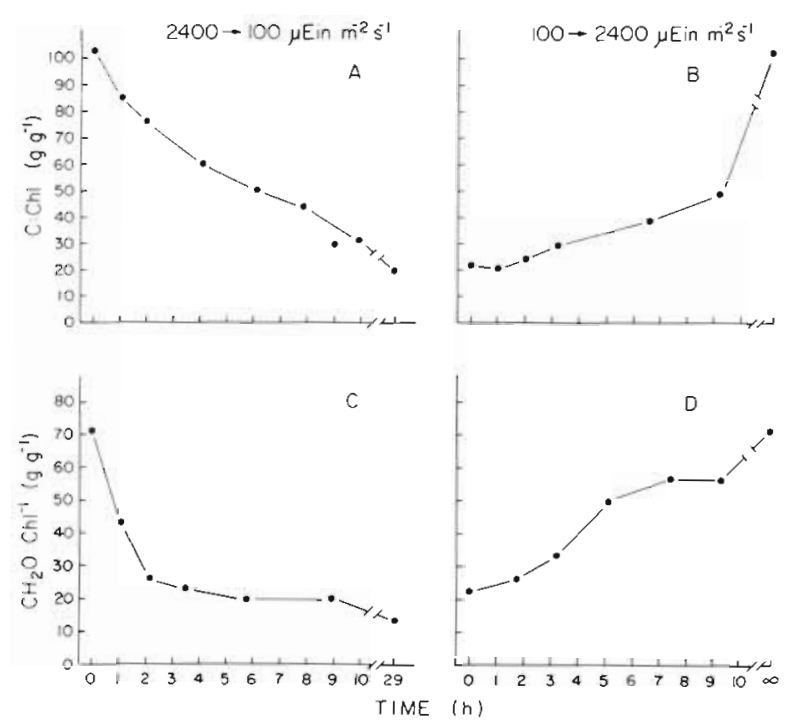

Fig. 3. Time course of photoadaptation of $(A, B)$ carbon: Chl and $(C, D)$ carbohydrate: $C h]$

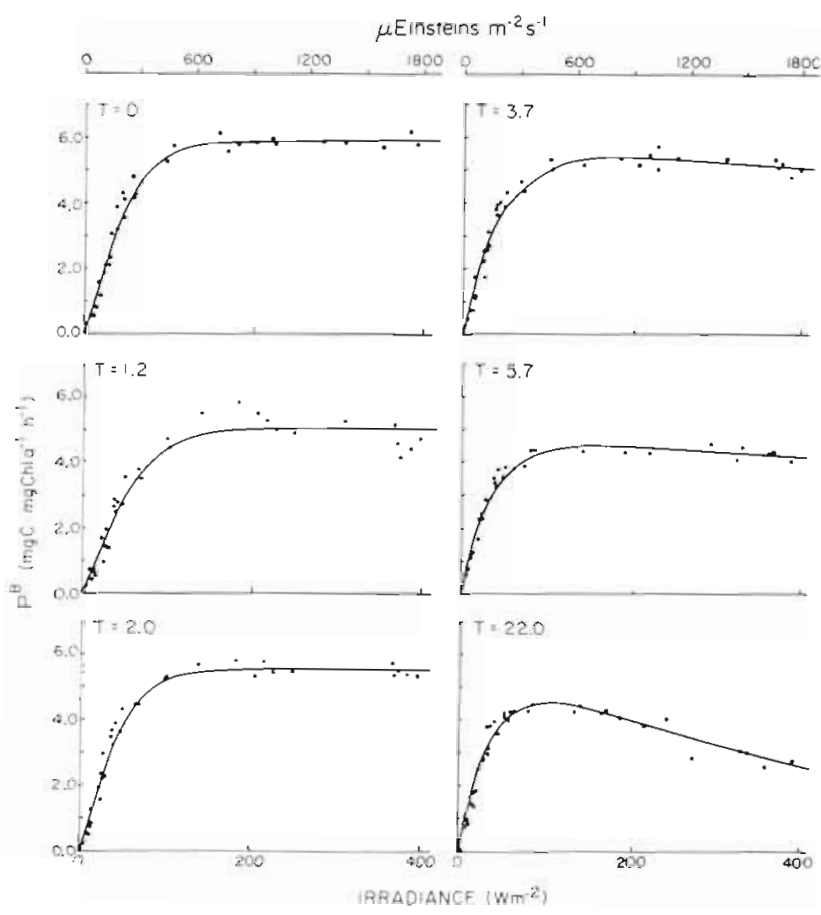

Fig. 4. Photoadaptation of photosynthesis-irradiance curves following an irradiance change from 2400 to $100 \mu \mathrm{Ein} \mathrm{m}^{-2} \mathrm{~s}^{-1}$

were duplicated, but not all measurements were made in each experiment.

The time-course of photoadaptation for reciprocal light-shifts between $100 \mu \operatorname{Ein} \mathrm{m}^{-2} \mathrm{~s}^{-1}$ (medium) and $2400 \mu$ Ein $\mathrm{m}^{-2} \mathrm{~s}^{-1}$ (high) is shown in Fig 3 to 7. Fluorescence yield, both in the absence and in the presence of DCMU decreased rapidly in response to a large increase in irradiance (Fig. 7 $\mathrm{A}_{i}$ cf. Loftus and Seliger, 1975; Heaney, 1978; Harris, 1980b; Vincent,

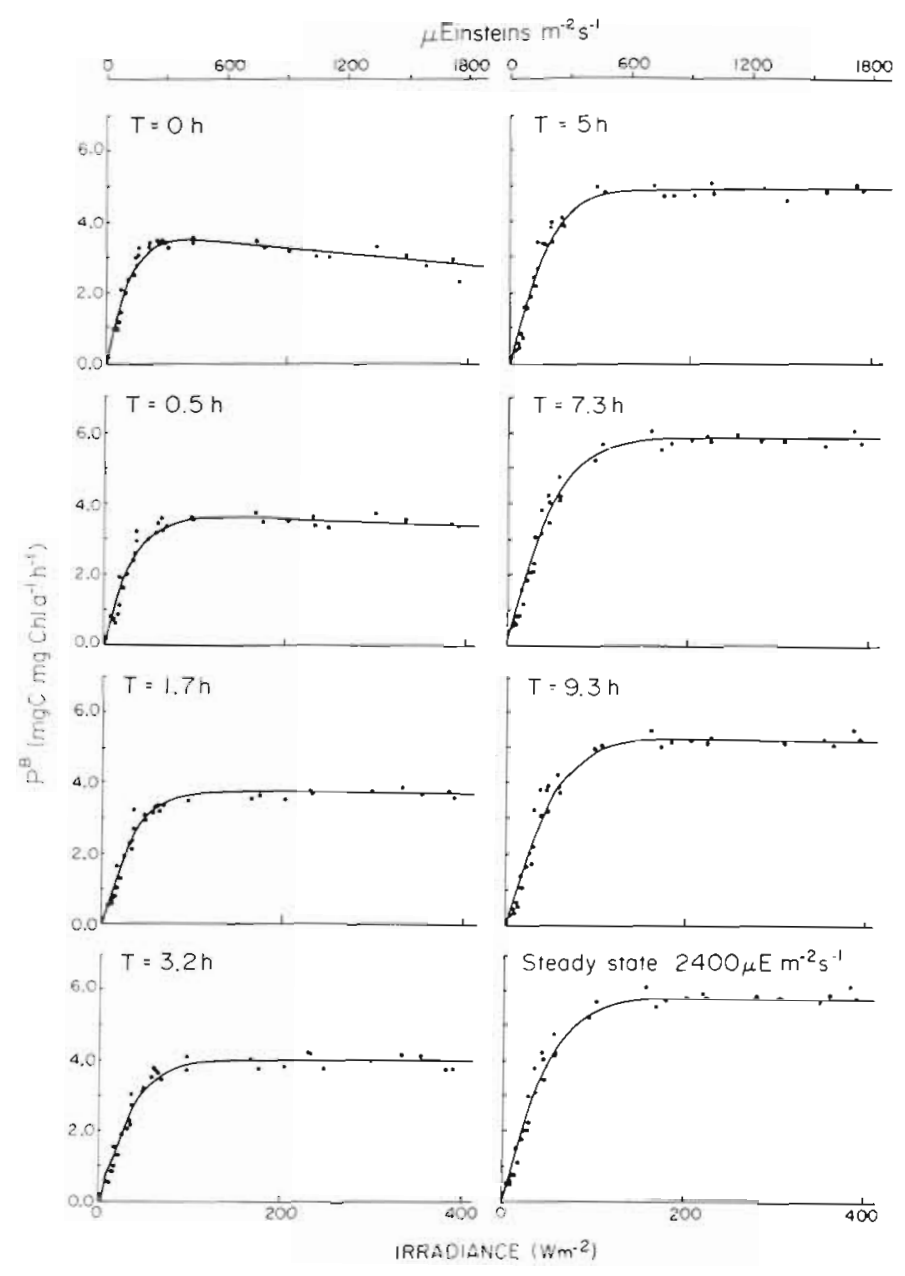

Fig. 5. Photoadaptation of photosynthesis-irradiance curves

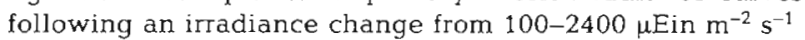

1980) with a temporal scale of less than $1 \mathrm{~h}$. The asymptote of the decline in fluorescence yield is not the same as the steady-state value for the higher light intensity because on the time-scale of days, photoinhibition of fluorescence is in large part a transient feature, but on the diurnal scale, fluorescence response can be considered as consistent with our model.

Chemical composition changēd more slowly than fluorescence: both $\mathrm{C}$ : chlorophyll (Fig. 3A, B) and carbohydrate:chlorophyll (Fig. 3C, D) changed to new levels over several hours, (cf. Gibson, 1978; Owens et al., 1980), with some indication that the rate of change for carbohydrate:chlorophyll was faster. Photosynthetic parameters, derived from photosynthesis-irradiance curves based on 20 min incubations (Platt et al., 1980; Lewis and Smith, 1983) (Fig. 4 and 5) showed variety of photoadaptive patterns:photosynthetic capacity, $\mathrm{P}_{\mathrm{m}}^{\mathrm{B}}$ responded over several hours, similar to $\mathrm{C}$ : chlorophyll (Fig. 6A, B; cf. Falkowski and Wirick, 1981); the initial slope of the photosynthesis-irradiance (P-I) curve, $\alpha$, 


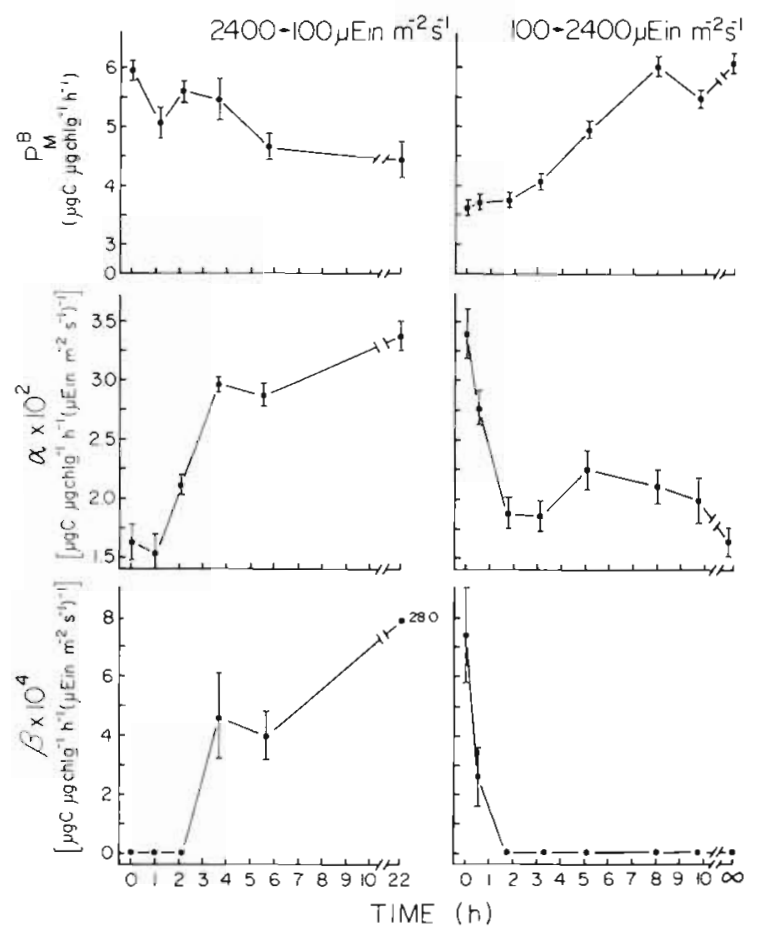

Fig. 6. Time-course of photoadaptation of the parameters of the P-I curves in Fig. 4 and 5

responded more rapidly than did $\mathrm{P}_{\mathrm{m}}^{\mathrm{B}}$ completing a transition in 2 to $4 \mathrm{~h}$ (Fig. 6C, D) and showed a temporal pattern similar to that of the fluorescence parameters, a relationship suggested by Welschmeyer and Lorenzen (1981). A term indicative of the degree of photoinhibition manifest in the P-I curve, $\beta$, showed a clearly discernable pattern (Fig. 6, E, F) that was not, however, symmetrical between reciprocal light-shifts as would be expected for a first-order reaction.

Results such as these must be corroborated by measurements on natural populations and generalized to include the effects of temperature, diurnal variation of irradiance, and species composition. This can be accomplished in part by performing light-switch experiments on the natural populations in situ (Tilzer and Goldman, 1978 and references therein; Gallegos et al., in press) and by measuring the change of the suite of photoadaptive parameters concurrent with vertical profiles of the same parameters. For example, an experiment on a diatom-dominated natural population from Bedford Basin (Fig. 7B) indicates that the rate constant for photoinhibition of fluorescence may be independent of temperature (compare with Fig. 7A) and can be used for interpreting vertical profiles of fluorescence from Bedford Basin during the spring bloom (Fig. 8).

To the extent that the model is applicable, the relationship expressed in Equation (8) (Fig. 1) implies that water columns which differ in physical scale should be

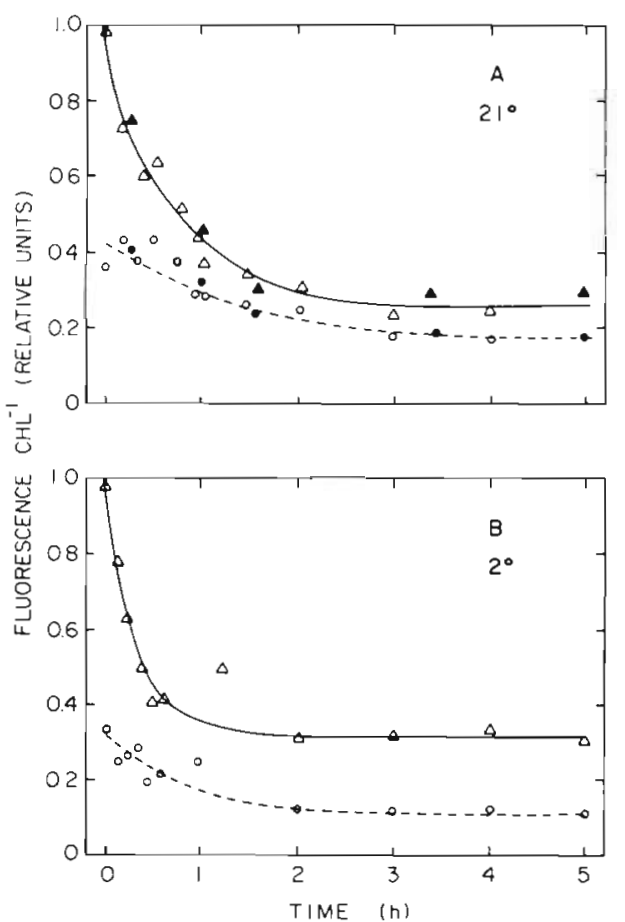

Fig. 7. Time-course of photoadaptation of fluorescence parameters following transfer from dim light to high light for (A) laboratory population at $21^{\circ} \mathrm{C}$, and (B) natural population at $2{ }^{\circ} \mathrm{C}$. Triangles represent DCMU poisoned samples

dynamically similar with respect to the importance of photoadaptation if the values of the dimensionless groups, $\mathrm{k}_{\mathrm{s}} \ell$ and $\mathrm{K}_{\mathrm{v}} / \gamma \ell^{2}$ are numerically identical. This provides a basis for scaling laboratory analogs such as

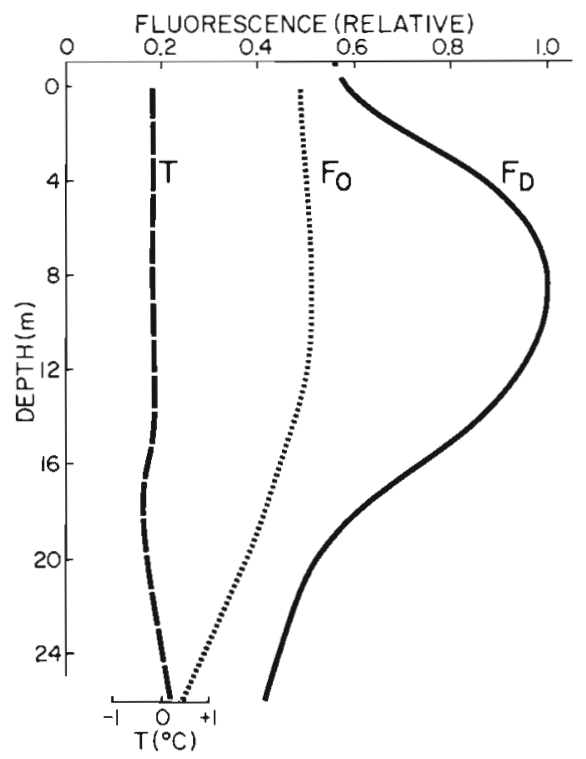

Fig. 8. Depth distribution of fluorescence $\left(F_{0}\right.$-without DCMU; $\mathrm{F}_{\mathrm{D}}$-with $\mathrm{DCMU}$ ) and temperature on $1 \mathrm{~d}$ during spring bloom. 16 March was characterized by winds in excess of $20 \mathrm{~m} \mathrm{~s}^{-1}$ with a correspondingly high rate of energy dissipation measured with a microscale velocity profiler 
microcosms (Platt, 1982). For example, microcosms, by definition, are smaller than the natural system being reproduced. If the photoadaptation process is considered important, then the reduction of $\ell$ in the microcosm must be accompanied by an increase in the optical attenuance and a rather severe decrease in $K_{v}$ since the mixing process scales as $\ell^{-2}$. Attempts to induce equivalent turbulent velocities in scaled down ecosystems as found in the natural system (Nixon et al., 1980) result in mixing rates over the equivalent optical length scale considerably higher than in the natural system. The use of such dimensionless groups in comparative studies can remove effects due principally to differences in scale.

In this paper, we have focussed on the distribution of photoadaptive properties with depth. An interesting question that follows is how variations in the parameters influence variations in primary production. Formally this would involve evaluating partial derivatives of photosynthesis with respect to the parameters. Since the relationship between photosynthesis and irradiance is time-dependent and non-linear, the influence of physiological adaptation on photosynthetic performance is potentially of some importance (Platt and Denman, 1975; Marra, 1978b; Falkowski and Wirick, 1981). Here, we have shown that the problem of assessing photosynthesis in vertically mixed water columns can be simplified: by determining the distributions of photoadaptive variables, one can determine the important scales for photoadaptation and design experiments accordingly.

Acknowledgements. We would like to thank Drs. C. L. Gallegos, R. Geider, E. P. W. Horne, W. G. Harrison, W. K. Li and J. C. Smith for numerous discussions and comments on the manuscript. Drs. M. J. Fasham, M. Levandowsky and K. H Mann also critically reviewed the manuscript and P. D. Lewis assisted with the analysis for which we are grateful. MRL was supported by the National Sciences and Engineering Research Council (Canada) and by a Dalhousie University Fellowship.

\section{LITERATURE CITED}

Abbott, M. R., Richerson, P. J., Powell, T M. (1982). In situ response of phytoplankton fluorescence to rapid variations in light. Limnol. Oceanogr. 27: 218-225

Auclair, J. C., Demers, S., Fréchette, M., Legendre, L., Trump, C. L. (1982). High frequency endogenous periodicities of chlorophyll synthesis in estuarine phytoplankton. Limnol. Oceanogr. 27: 348--352

Beardall, J, Morris, I. (1976). The concept of light intensity adaptation in marine phytoplankton: some experiments with Phaeodactylum tricornutum. Mar Biol. 37: 377-387

Brooks, J. E. (1964). Acclimation to light intensity in two species of marine phytoplankton, Dunaliella tertiolecta Butcher and Skeletonema costatum (Greve.) Cleve. M.S. thesis, University of Southern California

Cullen, J. C., Renger, E. (1979). Continuous measurement of the DCMU-induced fluorescence response of natural phytoplankton populations. Mar. Biol. 53: 13-20

Demers, S., Legendre, L. (1981). Mélange vertical et capacité photosynthétique du phytoplancton estuarien (estuaire de Saint-Laurent). Mar. Biol. 64: 243-250

Denman, K. L., Platt, T. (1975). Coherences in the horizontal distributions of phytoplankton and temperature in the upper ocean. Mém. Soc. R. Sci. Liège (6e sér.) 7: 19-30

Dera, J., Gordon, H. R. (1968). Light field fluctuations in the photic zone. Limnol. Oceanogr. 13: 697-699

Dera, J., Olszewski, J. (1967). On the natural irradiance fluctuations affecting photosynthesis in the sea. Acta Geophys. Polon. 15: 351-364

Derenbach, J. B., Astheimer, H., Hansen, H. P., Leach, H (1979). Vertical microscale distribution of phytoplankton in relation to the thermocline. Mar. Ecol. Prog. Ser. 1: $187-193$

Falkowski, P. G. (1980). Light-shade adaptation in marine phytoplankton. In: Falkowski, P. G. (ed.) Primary productivity in the sea. Plenum Press, New York, p. 99-119

Falkowski, P. G. (1981), Light-shade adaptation and assimilation numbers. J. Plankton Res. 3: 203-216

Falkowski, P. G. (in press). Light-shade adaptation and vertical mixing of marine phytoplankton: a comparative field study. J. mar Res. 41

Falkowski, P. G., Wirick, C. D. (1981). A simulation model of the effects of vertical mixing on primary productivity. Mar. Biol. 65: 69-75

Gallegos, C. L., Platt, T. (1982). Phytoplankton production and water motion in surface mixed layers. Deep Sea Res. 29: $65-76$

Gallegos, C. L., Platt, T., Harrison, W. G., Irwin, B. (in press) Photosynthetic parameters of Arctic marine phytoplankton: vertical variations and time scales of adaptation Limnol. Oceanogr

Gibson, C. E. (1978). Field and laboratory observations on the temporal and spatial variation of carbohydrate content in planktonic blue-green algae in Lough Neagh, Northern Ireland. J. Ecol. 66: 97-115

Harris, G. P. (1980a). Temporal and spatial scales in phytoplankton ecology. Mechanisms, methods, models, and management. Can. J. Fish. aquat. Sci. 37: 877-900

Harris, G. P. (1980b). The relationship between chlorophyll a fluorescence, diffuse attenuation changes and photosynthesis in natural phytoplankton populations. J. Plankton Res. 2: 109-127

Harris, G. P., Lott, J. N. A. (1973). Light intensity and photosynthetic rates in phytoplankton. J. Fish. Res. Bd Can. 30: $1771-1778$

Heaney, S. I. (1978). Some observations on the use of the in vivo fluorescence technique to determine chlorophyll $a$ in natural populations and cultures of treshwater phytoplankton. Freshwat. Biol. 8: 115-126

Hill, J. C. (1976). Homogeneous turbulent mixing with chemical reaction. Ann. Rev. Fluid Mech. 8: 135-161

Hitchcock, G. L. (1980). Influence of temperature on the growth rate of Skeletonema costatum in response to variations in daily light intensity. Mar Biol. 57: 261-269

Jewson, D. H., Wood, R. B. (1975). Some effects on integral photosynthesis of artificial circulation of phytoplankton through light gradients. Verh. int. Verein. Limnol. 19: 1037-1044

Jitts, H. R., McAllister, C. D., Stephens, K., Strickland, J. D. H. (1964). The cell division rates of some marine phytoplankters as a function of light and temperature. J. Fish. Res. Bd Can. 21: 139-157

Kiefer, D. (1973). Chlorophyll a fluorescence in marine centric 
diatoms: response of chloroplasts to light and nutrient stress. Mar. Biol. 23: 39-46

Kremer, J. N., Nixon, S. W. (1978). A coastal marine ecosystem: simulation and analysis. Springer Verlag, New York

Laws, E. A., Bannister, T. T. (1980). Nutrient- and lightlimited growth of Thalassiosira fluviatilis in continuous culture, with implications for phytoplankton growth in the ocean. Limnol. Oceanogr. 25: 457-473

Lewis, M. R., Smith, J. C. (1983). A small volume, shortincubation-time method for measurement of photosynthesis as a function of incident irradiance. Mar. Ecol. Prog. Ser. 13: $99-102$

Loftus, M. E., Seliger, H. H. (1975). Some limitations of the in vivo fluorescence technique. Chesapeake Sci. 2: 79-92

Lorenzen, C. (1966). A method for the continuous measurement of in vivo chlorophyll concentration. Deep Sea Res. 13: $223-227$

Marra, J. (1978a). Effect of short-term variations in light intensity on photosynthesis of a marine phytoplankter: a laboratory simulation study. Mar. Biol. 46: 191-202

Marra, J. (1978b). Phytoplankton photosynthetic response to vertical movement in a mixed layer. Mar. Biol. 46: 203-208

Marra, J. (1980). The time course of light intensity adaptation in a marine diatom. Mar. Biol. Lett. 1: 175-183

Nixon, S. W., Alonso, D., Pilson, M. E. Q., Buckley, B. A (1980). Turbulent mixing in aquatic microcosms. In: Geisy, J. P., Jr. (ed.) Microcosms in ecological research. U.S. Dept. Energy Symp. Ser. 52: NTIS CONF-781101

Owens, T G., Falkowski, P. G., Whitledge, T. E. (1980). Diel periodicity in cellular chlorophyll content in marine diatoms. Mar. Biol. 59: 71-77

Paasche, E. (1968). Marine plankton algae grown with lightdark cycles. II. Ditylum brightwellii and Nitzschia turgidula. Physiol. Plant. 21: 66-77

Platt, T. (1982). Thinking in terms of scale: introduction to dimensional analysis. In: Platt, T., Mann, K. H. Ulanowicz, R. E. (ed.) Mathematical models in biological oceanography. UNESCO Press, Paris, p. 112-121

Platt, T., Denman, K. L. (1975). A general equation for the mesoscale distribution in the sea. Mèm. Soc. R. Sci. Liège (6e sér.) 7: 31-42

Platt, T., Gallegos, C. L. (1980). Modelling primary production. In: P. G. Falkowski (ed.) Primary productivity in the sea. Plenum Press, New York, p. 339-362

Platt, T., Gallegos, C. L., Harrison, W. G. (1980). Photoinhibition of photosynthesis in natural assemblages of marine phytoplankton. J. mar. Res. 38: 687-701

Platt, T., Harrison, W. G., Irwin, B., Horne, E. P., Gallegos, C. L. (1982). Photosynthesis and photoadaptation of marine phytoplankton in the arctic. Deep Sea Res. 29: $1159-1170$

Pollard, R. T. (1977). Observations and models of the structure of the upper ocean. In: Kraus, E. B. (ed.) Modelling and prediction of the upper layers of the ocean. Pergamon, Oxford, p. 102-117

Prézelin, B. B., Matlick, H. A. (1980). Time-course of photoadaptation in the photosynthesis-irradiance relationship of a dinoflagellate exhibiting photosynthetic periodicity. Mar Biol. 58: 85-96

Prézelin, B. B., Sweeney, B. (1977). Characterization of photosynthetic rhythms in marine dinoflagellates. Plant Physiol. 60: 388-392

Rivkin, R. B., Seliger, H. H., Swift, E., Biggley, W H. (1982). Light-shade adaptation by the oceanic dinoflagellates Pyrocystis noctiluca and $P$. fusiformis. Mar. Biol. 68: 181-191

Steemann Nielsen, E., Hansen, V. K. (1959). Light adaptation in marine phytoplankton populations and its interrelation with temperature. Physiol. Plant. 12: 353-370

Steemann Nielsen, E., Hansen, V. K., Jorgensen, E. G. (1962). The adaptation to different light intensities in Chlorella vulgaris and the time dependence on transfer to a new intensity. Physiol. Plant. 15: 505-517

Steemann Nielsen, E., Jorgensen, E. G. (1968). The adaptation of plankton algae III. With special consideration of the importance in nature. Physiol. Plant. 21: 647-654

Talling, J. F. (1966). Photosynthetic behavior in stratified and unstratified lake populations of a planktonic diatom. J. Ecol. 54: 99-127

Tilzer, M. M., Goldman, C. R. (1978). Importance of mixing thermal stratification and light adaptation for phytoplankton productivity in Lake Tahoe (California-Nevada). Ecology 59: 810-821

Vincent, W. F. (1979). Mechanisms of rapid photosynthetic adaptation in natural phytoplankton communities. I. Redistribution of excitation energy between photosystems I and II. J. Phycol. 15: 429-434

Vincent, W. F. (1980). Mechanisms of rapid photosynthetic adaptation in natural phytoplankton communities. II Changes in photochemical capacity as measured by DCMU-induced chlorophyll fluorescence. J. Phycol. 16 568-577

Welschmever, N. A., Lorenzen, C. J. (1981). Chlorophyllspecific photosynthesis and quantum efficiency at subsaturating light intensities. J. Phycol. 17: 283-293

Woods, J., Onken, R. (1982). Diurnal variation and primary production in the ocean - preliminary results of a Lagrangian ensemble model. J. Plankton Res. 4: 735-756

Wroblewski, J. S., O'Brien, J. J., Platt, T (1975). On the physical and biological scales of phytoplankton patchiness in the ocean. Mém. Soc. R. Sci. Liège 7: 43-57

Yentsch, C. S., Lee, R. W (1966). A study of photosynthetic light reactions and a new interpretation of sun and shade phytoplankton. J. mar. Res. 24: 319-337

Zaneveld, J. R., Kitchen, J. C., Pak, H. (1981). The influence of optical water type on the heating rate of a constant depth mixed layer. J. geophys. Res. 86: 6426-6428 